\title{
Study on Automatic North-Seeking Key Technologies of Maglev Gyroscope
}

\author{
Shi Zhen", Yang Zhiqiang and Zhang Zhe \\ Institute of Geology Engineering and Geomantics, Chang'an University, Xi'an, 710054, China
}

\begin{abstract}
First, this paper proposes a static north-seeking mode based on the meridian seeking torque of gyroscope. We analyze the force situation and stability of the gyro magnetic suspension system in this mode and focus on the principle of torque balance feedback control technology. Two-position rotary control technology has been designed based on the sensitive state of the gyro rotor in four special north-seeking positions to reduce the impact of the systematic errors on the single position north-seeking. Later, we use the north-seeking data to simulate the real-time north-seeking process. Meanwhile, filter processing is used to improve the quality of the result. Finally, a comprehensive inspection is made to test the key technologies of the maglev gyro through the practice of an underground project. And it proves that these technologies have a favorable effect in practical applications.
\end{abstract}

Keywords: Maglev gyroscope, orientation survey, meridian seeking torque, rotary control.

\section{INTRODUCTION}

The traditional suspension gyrotheodolite (GAK-1, JT15) is the directional instrument which is commonly used in mining through measurement. In actual operation, it's necessary to manually suspend gyro and track gyro swing and is finally used to calculate the azimuth of the measuring line through the transit method or the amplitude method. The entire operation has more human intervention and the observation errors seriously affect the final orientation result.

With the fast development of technologies in surveying, it is very popular to develop new automated, high precision gyrotheodolite (total station) at home and abroad. Development of the new gyrotheodolite (total station) can be summarized as the following two aspects: First, developing new gyro suspension technologies, such as flexible gyro, ESG (electrically suspended gyroscope), laser gyro, etc; Second, developing new technologies (modern optical, modern electrical machinery, etc) based on the traditional suspension gyrotheodolite to improve the quality of data sampling and data processing. For example, the Gyromat2000 gyrotheodolite of Germany develops step method to find the north coarsely and integral method to find the north accurately. It is internationally recognized as the best gyrotheodolite with an accuracy of 3 to 5 seconds.

GAT high-precision maglev gyro total station is developed by Chang'an University together with the sixteenth Institute of China Aerospace Science and Technology Corporation. The instrument has an accuracy of better than 5 second with China's independent intellectual

*Address correspondence to this author at the Institute of Geology Engineering and Geomantics, Chang'an University, Xi'an, 710054, China; Tel/Fax:+86-029-82339032; E-mail: shinsar_gps@163.com property right. The instrument using magnetic suspension technology and a number of advanced technologies makes the directional accuracy of the gyro stable and easy to achieve.

\section{KEY TECHNOLOGIES OF MAGLEV GYRO}

At present, most of the gyrotheodolites (gyro total station) are using the wire or tape suspension technology. The properties of the tape (elastic limit, tensile strength, elastic after effect, corrosion resistance, temperature coefficient of the elastic modulus as well as magnetic field) will directly affect the north-seeking accuracy of the gyro. In addition, the close link between the suspension and the gyro sensitive part will reduce the accuracy of the north-seeking result. Therefore, all the wire or tape suspension gyros need the zero observation to find the balance location of the wire or tape.

It is the purpose of all north-seeking gyroscopes to determine the meridian of the station. There is a reciprocating motion of the gyro rotation axis near the meridian of the station. The meridian of the station is constantly changing with the Earth rotation. Meanwhile, the swing center of the gyro rotation axis is constantly changing. We can say that the north-seeking process of the gyro is the process of the gyro rotation axis tracking the meridian [1]. So the gyroscope can also be defined as meridian tracking measurement system. The gyro can finish the orientation survey through tracking the meridian dynamically (Fig. 1).

The high-speed rotation gyro generates a meridian seeking torque $M$ due to the Earth rotation. The torque $M$ changes constantly with the gyro rotation axis along the meridian. The torque $M$ is:

$M=H \times \omega_{e} \cos \phi \sin \alpha$ 


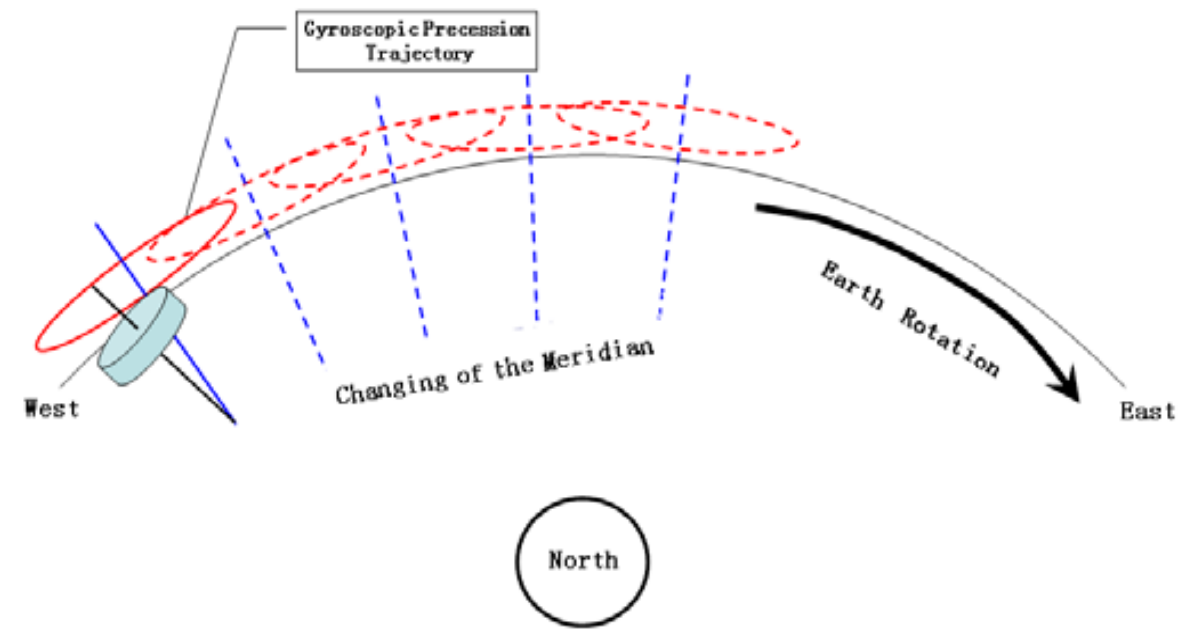

Fig. (1). Analysis of the north-seeking trajectory.

If we apply an opposite and equal torque $M^{\prime}$ upon the gyroscope sensitive part in horizontal direction, the sensitive part will be in torque equilibrium and the rest in a certain position. By measuring the torque $M^{\prime}$, we can get the deflection angle $\alpha$ of the gyro rotation axis according to the Eq. (1). The angle $\alpha$ is:

$$
\alpha=\arcsin \left(\frac{M^{\prime}}{H \times \omega_{e} \cos \phi}\right)
$$

Then we can get the true north direction according to the equilibrium direction of the gyro rotation axis. The maglev gyro can finish the orientation survey through tracking the meridian statically.

\subsection{Magnetic Suspension Technology}

It is important to reduce the close link of the gyro sensitive part with the outside. The maglev gyro allows the gyro sensitive part to make a north-seeking process in a non-interference environment. Fig. (2) is a diagram of the design principle of the magnetic suspension system. When the north-seeking process begins, the magnetic suspension coil is energized and the generated magnetic force will let the armature and the gyro sensitive part suspend; After the measurement the magnetic suspension coil is cut, magnetic force disappears, and the gyro sensitive part automatically descends [2].

\subsubsection{Force Analysis of Magnetic Suspension System}

Through the analysis of the gyro sensitive part during the north-seeking, we come to know that the Earth surface will ascend relative to decline of the gyro rotation axis because of the Earth rotation. There will be a slight declination between the rotation axis and the Earth surface. It will generate a precession torque making the gyro sensitive part move to the meridian and change the force factors of the gyro sensitive part in vertical direction. The calculation equation is:

$F_{\mathrm{d}}=b \dot{x}+J \ddot{x}$

$x$ is the offset of the gyro sensitive part. According to the Eq. (3) extra forces are subjected to the gyro sensitive part besides the gravity caused by the offset $x$. The offset is related to the distance of the gyro sensitive part deviated from the equilibrium position. We conclude that $x$ is related to the deflection angle of the gyro rotation axis deviated from the meridian. The bigger angle generates the bigger $x$. In this state the force equation of the gyro sensitive part in vertical direction is:

$$
F_{M}-m g-b \dot{x}=J \ddot{x}
$$

where: $F_{\mathrm{M}}$-magnetic force of the inductance coil applied to the gyro sensitive part; $m$-mass of the gyro sensitive part; $b$-damping coefficient; $J$-rotational inertia of the gyro sensitive part; $x$ - distance of the gyro sensitive part deviated from the equilibrium position in vertical direction

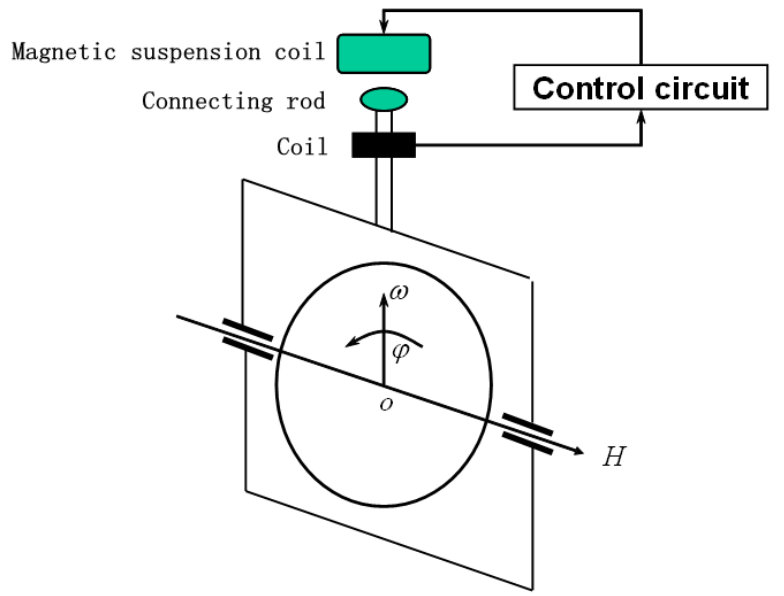

Fig. (2). Maglev system diagram.

\subsubsection{Stability Technology of the Magnetic Suspension System}

The north-seeking state of the magnetic suspension system is directly related to the accuracy of the north-seeking result, long-term and short-term stability of the instrument constant and some other key technical indicators. In order to keep the system in a good state, structure optimization is conducted from the following aspects: 
First, the locking technology ensures the stability of the magnetic suspension system in non-working state. The locking system is made up of inductance coil, spring, and sheet. It can fix the gyro sensitive part firmly in the rotary housing in non-working state. There is a pressure of $50 \mathrm{~N}$ to guarantee that the magnetic suspension system will not be damaged by transportation or other reasons.

Second, the stable slot ensures the gyro sensitive part rising up and falling down in the same position. The inverted cone at the bottom of the gyro sensitive part fits together with the stable slot to ensure the gyro sensitive part rising up and falling down in the same position.

Third, micro-torque and stability control system of the conductive hairspring are applied. The conductive hairspring is the only active contact of the gyro sensitive part with the outside. And it is the only medium for the high-speed gyro motor to exchange electric signal with the outside. In order to reduce the impact of the conductive hairspring on the gyro sensitive part, the system contains two pieces of conductive hairspring in parallel to reduce the impact of the micro torque. And the conductive hairspring is made up of stable bronze wire to make sure the stability of the micro torque.

In addition, a multi-layer magnetic shielding technology is used to avoid the influence of the external electromagnetic field.

Compared with wire or tape suspension gyro, the magnetic suspension gyro replaces the tedious process of manual handling and observing with the help of magnetic suspension coils. And it will need no zero observation. Because gyro sensitive part is in contact with the suspended part in the north-seeking process, the friction interference problems brought about by the traditional mechanical suspension technologies disappear. It will extend the life of the gyroscope.

\subsection{Torque Balance Feedback Control Technology}

\subsubsection{Angular Displacement Calculation Based on the Reverse Torque}

As shown in Fig. (3), the gyro sensitive part is in a suspended state. The Earth rotation produces a meridian seeking torque $M$ and it will make the gyro rotation axis approximate to the meridian of the station.

On the impact of $M$, the gyro rotation axis will show a precession effect around the meridian plane. Then a reverse torque $M^{\prime}$ is applied to the gyro sensitive part. Let $M^{\prime}=M$, the gyro sensitive part will be in a static state. By measuring the reverse torque $M^{\prime}$ we can get the deflection angle of the gyro rotation axis according to the Eq. (1).

\subsubsection{Detection and Control of the Torque Balance}

It is a dynamic process of adjustment for the gyro sensitive part from starting to the torque balance state. At the beginning the reverse torque calculated from the stator and rotor current value can not reflect the meridian seeking torque of the gyro sensitive part correctly, because the gyro sensitive part is in an unstable state. Thus the stability of the gyro sensitive part is detected before collecting data from the stator and rotor current.

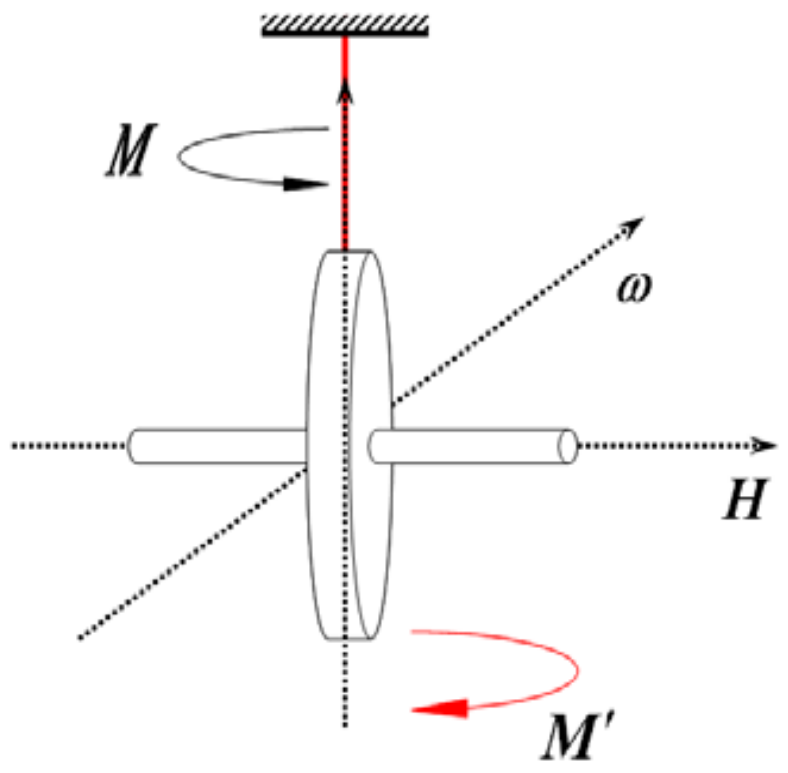

Fig. (3). Maglev gyro north measurement schematic.

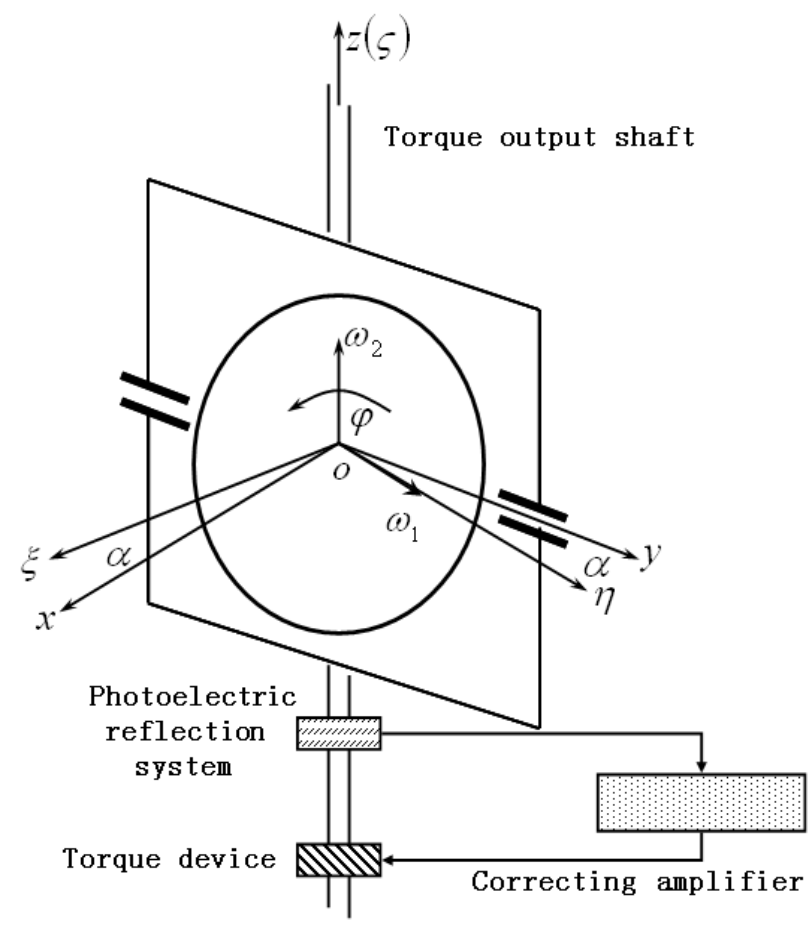

Fig. (4). Photoelectric torque feedback control principle.

The transmitters and receivers of the photoelectric sensor point at the reflecting prism group when the gyro sensitive part is in a suspension state. After the transmitters send incident light to the reflecting prism group, the receiver can catch the reflected light and the photoelectric sensor calculates the angular displacement of the gyro sensitive part according to positions of the incident light and the reflected light. Then the angular displacement is converted to electrical signal. The electrical signal is converted to digital 
data after the process of AC-amplification, demodulation, DC-amplification, low-pass filtering, analog conversion and so on. Then the digital data is converted to analog signal and sent to the torque compensator. The gyro sensitive part can achieve a balance state after the compensator adjusts the current and changes the reverse torque (Fig. 4).

\subsection{Two-Position Rotary Control Technology}

The technology of two-position rotary control is to calculate the north declination of the gyro rotation axis accurately according to the north-seeking data of two different positions. Theoretically, we can get the accurate direction of the true north after the rough north-seeking process and the first accurate north-seeking process. But it contains some systematic errors, such as eccentric error of the horizontal angle measurement system, constant drift error of the torque balance feedback control device and so on. These systematic errors show systematic in short-term or single measurement and show contingent in long-term or multiple measurements. Therefore another accurate northseeking process is added after one rough north-seeking process and one accurate north-seeking process to eliminate the impact of the systematic errors.

Fig. (5) is the function relationship diagram of the meridian seeking torque and the north declination of the gyro rotation axis. Suppose the gyro rotation axis points at A position with a declination of $\alpha$ after the rough northseeking process. Then $B$ is in symmetrical position of $A$, and C is opposite to B, and D is opposite to A. According to Eq. (1) the meridian seeking torque of the gyro at position A, B, C, D is:

$$
\begin{aligned}
& M_{A}=H \times \omega_{e} \cos \phi \sin \alpha_{0} \\
& M_{B}=H \times \omega_{e} \cos \phi \sin \left(-\alpha_{0}\right)=-M_{A} \\
& M_{C}=H \times \omega_{e} \cos \phi \sin \left(180^{\circ}-\alpha_{0}\right)=M_{A} \\
& M_{D}=H \times \omega_{e} \cos \phi \sin \left(180^{\circ}+\alpha_{0}\right)=-M_{A}
\end{aligned}
$$

Suppose $M_{f}$ is the systematic interference torque of the gyro sensitive part. Then the actually measured torque of the gyro rotation axis in position $\mathrm{A}$ is:

$$
M_{1}=M_{A}+M_{f}
$$

If the second accurate north-seeking process is conducted at position $\mathrm{B}$, the difference of the two accurate northseeking processes will be small and the second accurate north-seeking process will lose its meaning because position $\mathrm{A}$ is very close to position B after the rough north-seeking process.

If the second accurate north-seeking process is conducted at position $\mathrm{C}$, the meridian seeking torque of the position $\mathrm{C}$ is the same as the meridian seeking torque of the position A but the gyro rotation axis has rotated nearly $180^{\circ}$, and the systematic interference torque of the gyro sensitive part will be $-M_{f}$. Then the actually measured torque of the gyro rotation axis at position $\mathrm{C}$ is:

$M_{2}=M_{A}-M_{f}$

According to Eq. (5) and Eq. (7), the north declination of the gyro rotation axis is:

$$
\alpha=\arcsin \left(\frac{M_{1}+M_{2}}{2 \cdot H \times \omega_{e} \cos \phi}\right)
$$

If the second accurate north-seeking process is conducted at position $\mathrm{D}$, the meridian seeking torque of the position $\mathrm{D}$ is opposite to the meridian seeking torque of the position A. And the gyro rotation axis has rotated $180^{\circ}$ which will eliminate the eccentricity error of the horizontal angle measurement system. The systematic interference torque of the gyro sensitive part will be $M_{f}$. Then the actually measured torque of the gyro rotation axis at position $\mathrm{D}$ is:

$M_{2}=-M_{A}+M_{f}$

According to Eq. (5) and Eq. (8), the north declination of the gyro rotation axis is:

$\alpha=\arcsin \left(\frac{M_{1}-M_{2}}{2 \cdot H \times \omega_{e} \cos \phi}\right)$

According to Eq. (11) and Eq. (13) the difference of the two methods to calculate the north declination of the gyro rotation axis is about the interference torque. More studies are needed to know more characters of the torque $M_{f}$,
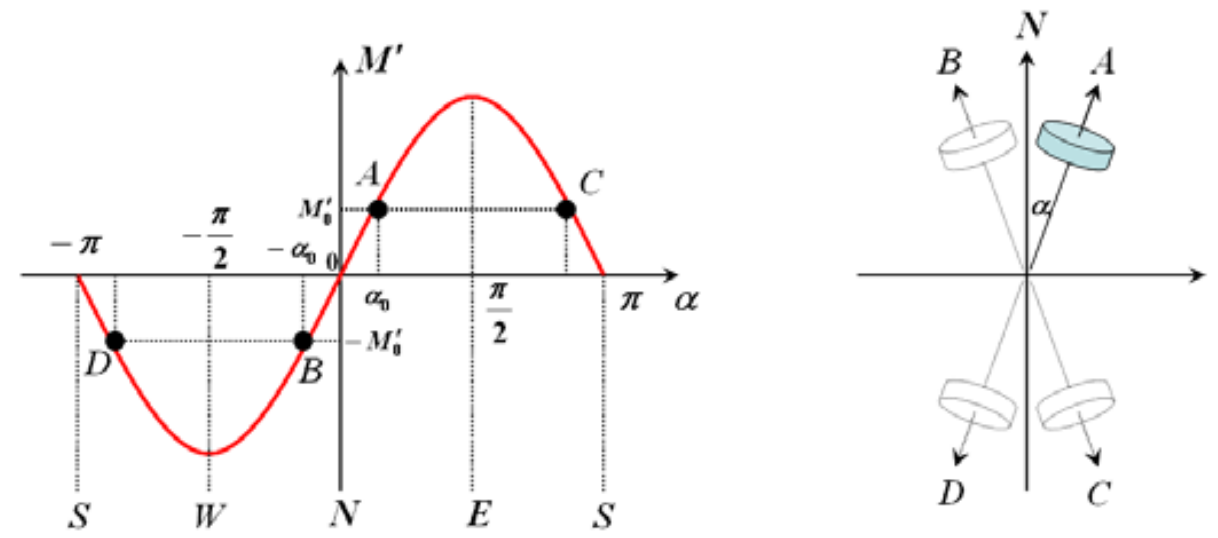

Fig. (5). Two-position north-seeking principle. 
because the structure of the maglev gyro is very complicated and the external environmental impact is uncertain. In practical application we prefer to choose position $\mathrm{D}$ as the second accurate north-seeking position and choose Eq. (13) to calculate the north declination of the gyro rotation axis for the following reasons:

First, position D is opposite to position A. It is consistent with the observation principle and conducive to eliminate the eccentric error.

Second, the meridian seeking torque of position D is opposite to the meridian seeking torque of position A. The torque balance control device applies two opposite torques in the two accurate north-seeking processes. It is conducive to eliminate the systematic errors.

\subsection{Data Collection and Real-Time Simulation Technology}

When the gyro sensitive part is in a stable state, the torque device begins to collect data and calculate the meridian seeking torque. The bigger the north declination $\alpha$ of the gyro rotation axis is, the bigger the meridian seeking torque $M$ of the gyro sensitive part will be. And bigger current and bigger reverse torque $M^{\prime}$ are needed to keep the sensitive part stable. There is a certain mathematical relationship between the current and the reverse torque. The rotor current is $I_{R}$, the stator current is $I_{S}$, and the reverse torque is:

$$
M^{\prime}=k \cdot I_{R} \cdot I_{S}
$$

where: $k$ is the coefficient of the torque device. In the northseeking process the torque device collects massive northseeking data to form the rotor and stator observation sequence. (As shown in Fig. (6), the blue is for the first north-seeking position data and red is for the second northseeking position data.) By the method of calculating average value of the data we get the reverse torque:

$$
M^{\prime}=\frac{k \cdot \sum_{i=1}^{n} I_{R i} \cdot I_{S i}}{n}
$$

where: $n$ is the number of the data.

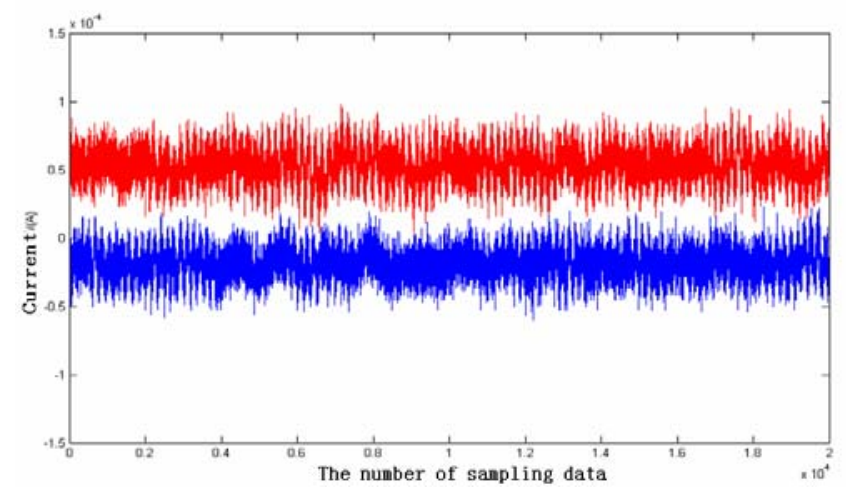

Fig. (6). Maglev gyro observation sequence.

\section{APPLICATION OF MAGLEV GYRO IN PRACTICE}

Due to the limitation of the environment, it is difficult to finish the orientation survey by GPS or other methods in the urban subway construction, mining tunnel through or other underground projects. Connection measurement and traverse are used to transfer bearings to the underground. But the accumulation of the errors will affect the quality of the underground project. Therefore gyroscope is used in orientation survey of underground project. Since the gyroscope is sensitive to the angular momentum of the Earth, it is very independent. It is effective to reduce the limitation of the underground environment and the accumulation of the errors.

Take the following example to describe the practical application of the maglev gyro in a tunnel project. The tunnel is designed $5.2 \mathrm{~km}$ long and $460 \mathrm{~m}$ deep. Shaft excavation is used because of the geological condition with the method of one-way boring. (From one shaft to another) In order to keep the final breakthrough accuracy under $5 \mathrm{~cm}$ gyro orientation survey is conducted after the distance of 3.5 $\mathrm{km}$ long. The orientation survey results are as follows.

In the Table 1, the stability of sampling data is calculated by:

$m=\sqrt{\frac{\left[v_{1_{i}} v_{1_{i}}\right]+\left[v_{2_{i}} v_{2_{i}}\right]}{40000-1}}$

where: [ ] - sum of the data; $v_{1_{i}}=I_{1_{i}}-\bar{I}_{1}$ (Current of data $\mathrm{i}$ average current); $v_{2_{i}}=I_{2_{i}}-\bar{I}_{2}$ (Current of data $\mathrm{i}$ - average current).

From the Table 1 we can see the data of NO.3 is affected by the vehicle vibration and the stability of sampling data is bad. It is also reflected in the diagram of the sampling data in time sequence. And there is a large difference between the third gyro azimuth and the other three one. So we calculate the north-seeking result without the data of NO.3. Take the stability of the sampling data as the weight, we can get the final azimuth:

$\bar{\alpha}=\frac{P_{1} \alpha_{1}+P_{2} \alpha_{2}+P_{4} \alpha_{4}}{P_{1}+P_{2}+P_{4}}$

According to the calculating result the underground traverse is amended and finally the tunnel is successfully through with a breakthrough accuracy of less than $3 \mathrm{~cm}$.

Since the invention of the maglev gyro total station, it has been widely applied to South-to-North Water Diversion Project of China, Jinping hydropower tunnel, water conveyance tunnel of Qinling Taibai mountain area and so on. It has been used in more than 20 large underground projects, and achieved good effect.

\section{CONCLUSION}

(1) The use of magnetic suspension technology on GAT maglev gyro total station reduces the close link of the 
Table 1. Orientation Survey Results

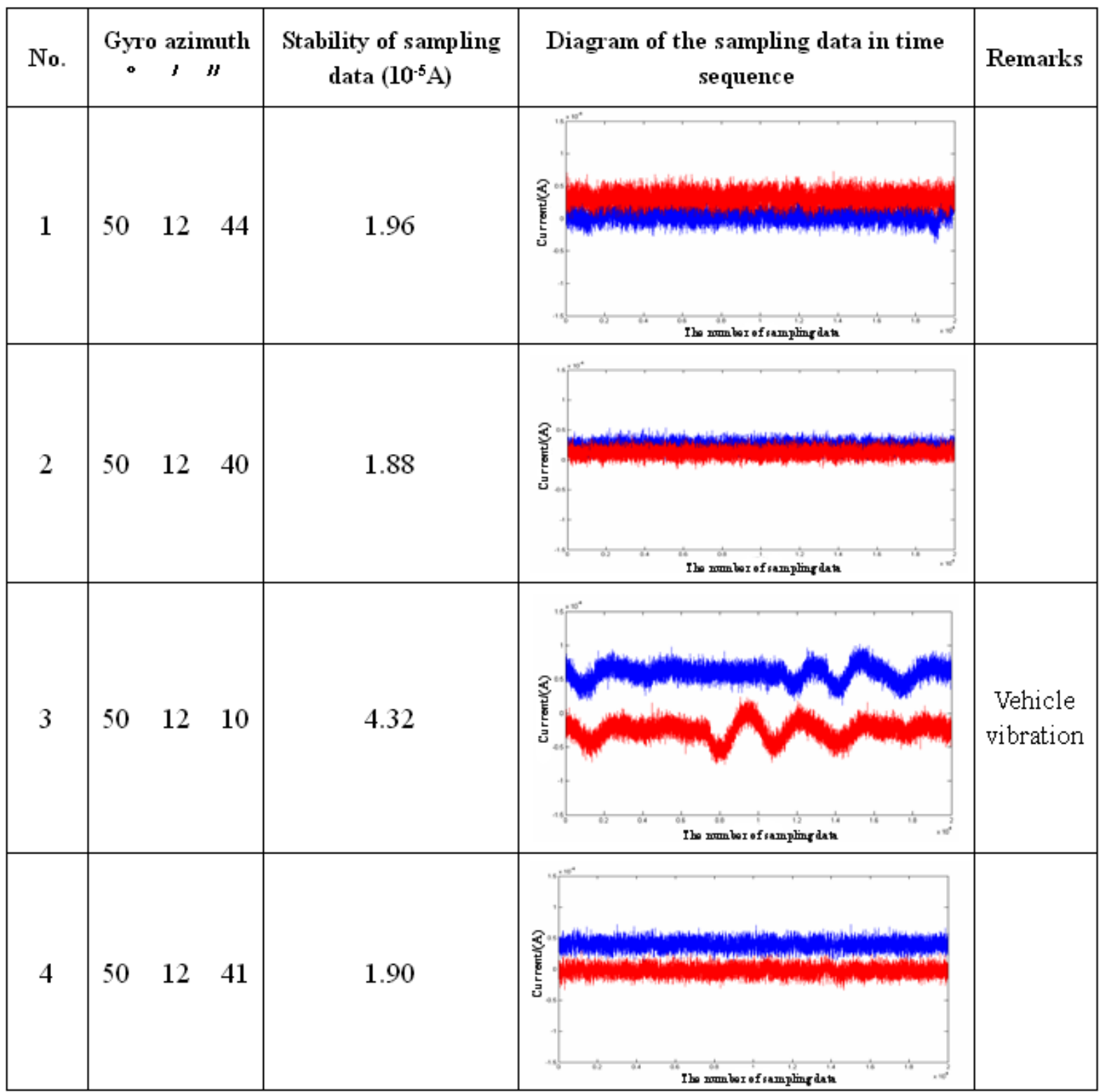

gyro sensitive part with the outside world, reduces the adverse effects of outside interference torque, improves the north-seeking accuracy and extends the use life of the instrument;

(2) GAT maglev gyro total station uses the reverse torque measurement in static state to replace the traditional measurement method. The north-seeking is no longer dependent on observation of the swing cycle of the gyro. It can collect huge amounts of data instantaneously. Post-processing is available to improve the north-seeking result.
(3) GAT maglev gyro total station uses the two-position rotary control technology, weakens the north-seeking systematic errors of traditional gyroscope and reduces the effect of disturbance torque.

(4) GAT maglev gyro total station achieves the data visualization processing through data simulation technology. Filtering process can also be used to improve the north-seeking result.

\section{CONFLICT OF INTEREST}

The authors confirm that this article content has no conflict of interest. 


\section{ACKNOWLEDGEMENTS}

Declared none.

\section{REFERENCES}

[1] G.-L. Zhang, Mining Surveying [M], Beijing: China Mining University Press, 2005, pp. 15-20.

[2] Y. Zhang, Gyro suspension system [M], Shanghai: Shanghai Jiao Tong University Press, 1987.

Received: August 13, 2013

(c) Zhen et al.; Licensee Bentham Open.

This is an open access article licensed under the terms of the Creative Commons Attribution Non-Commercial License (http://creativecommons.org/licenses/by-nc/3.0/) which permits unrestricted, non-commercial use, distribution and reproduction in any medium, provided the work is properly cited. 